\title{
EDITORIAL
}

\section{ATENCIÓN FARMACÉUTICA, ESTUDIOS SOBRE USO DE MEDICAMENTOS Y OTROS}

\author{
Antonio Iñesta García
}

Escuela Nacional de Sanidad. Instituto de Salud Carlos III. Ministerio de Sanidad y Consumo.

En este número de la Revista se publican una serie de trabajos que tratan sobre medicamentos, pero en unos casos se podrían clasificar como estudios de utilización de medicamentos, en otros de investigación de servicios sanitarios, de evaluación de servicios sanitarios, o bien de estudio de resistencias en bacterias de origen animal. Ante la dificultad de englobarlos todos con una misma denominación, se opta por el título del editorial.

A principios de los noventa Hepler y Strand dieron lugar a la conceptualización de la Atención Farmacéutica (AF) como «La provisión responsable de farmacoterapia con el propósito de obtener resultados definidos que mejoren la calidad de vida de los pacientes» ${ }^{1}$. En este modelo los proveedores de atención farmacéutica (los farmacéuticos) en cooperación con los pacientes y médicos, mejoran los resultados de la farmacoterapia al prevenir, o detectar y resolver los problemas relacionados con medicamentos $^{2,3}$ (PRM), antes que estos den lugar a morbilidad y mortalidad relacionadas con medicamentos.

Hepler y Strand siguieron caminos diferentes y dieron lugar a dos formas de practica de la AF: Atención Farmacéutica orienta-

Correspondencia:

Dr. Antonio Iñesta García

Escuela Nacional de Sanidad

Instituto de Salud Carlos III

C/ Sinesio Delgado 8

28029 Madrid

Correo electrónico: ainesta@isciii.es da a enfermedades crónicas de Hepler ${ }^{4}$, y la Atención Farmacéutica Global de Strand ${ }^{3}$. La primera orientada a situaciones crónicas y la segunda a una aplicación sistemática en todo tipo de situaciones.

La forma de concebir la AF por Strand y colaboradores, dio lugar a un proyecto de investigación orientado a la práctica, denominado Proyecto Minnesota de investigación de Atención Farmacéutica (Minnesota Pharmaceutical Care Project) ${ }^{5,3}$. El resultado de la intervención con AF fue que el $70 \%$ de los pacientes encuentran mejoría, y el $30 \%$ restante se encuentra igual o peor. Uno de los resultados más positivos del proyecto fue el cambio de actitud del colectivo médico, que acabó considerando al farmacéutico como un componente complementario totalmente necesario en el equipo de salud, y no como una interferencia.

Hepler y sus colaboradores desarrollaron estudios de investigación con el nombre de proyectos TOM $^{6}$ (Therapeutic Outcomes Monitoring, Monitorización de Resultados Terapéuticos), usando el asma como prototipo (TOM-asthma en EE.UU. ${ }^{6}$ ). Otros estudios también sobre el asma se han realizado en Europa (Dinamarca ${ }^{7,8}$, Finlandia ${ }^{9}$, Alemania ${ }^{10}$, otros países europeos, y un proyectado meta-análisis EUROTOM -ASMA).

Aplicando un modelo u otro de AF se han llevado a cabo otros muchos estudios de investigación, los cuales trataban de demostrar una mejoría de la calidad de vida en pacientes con insuficiencia cardiaca ${ }^{11}$, diabetes ${ }^{12-14}$, 
hipertensión $^{15-18}$, hipercolesterolemia ${ }^{19,20}$, etc. Entre ellos uno de provisión de AF comunitaria en pacientes ancianos realizado en siete países $^{21}$. Los resultados más relevantes fueron: ahorro de costes y aumento de calidad de vida relacionada con la salud en determinados países; los pacientes del grupo intervención manifestaron mejor control de sus enfermedades y altos niveles de satisfacción, y los farmacéuticos y médicos tuvieron una opinión positiva del nuevo abordaje.

El estudio TOMCOR, cuyo artículo aparece en este número de la Revista, ha sido realizado en España para evaluar los efectos sobre pacientes coronarios de la nueva forma de actuar en AF frente al modelo tradicional. Es un estudio prospectivo con grupos de intervención y control, y asignación aleatoria de las farmacias a los grupos. Las farmacias no constituyen una muestra representativa de las farmacias españolas, y el estudio sólo puede evaluar los resultados de este programa de AF sobre pacientes coronarios.

Los resultados clínicos evidenciaron una disminución en la tasa anual de reinfartos en el grupo intervención, aunque la diferencia con el grupo control no era estadísticamente significativa, y una menor utilización de recursos sanitarios. En calidad de vida hubo una mejoría significativa en una de las dimensiones del cuestionario SF-36 (función física), aunque hubo empeoramiento en otras (función social, emocional y salud mental), probablemente por mayor conciencia de enfermedad. También obtuvieron mejoras significativas en el conocimiento sobre los factores de riesgo de la enfermedad coronaria, sobre la medicación y en la satisfacción con el servicio.

Un meta-análisisde los resultados de 95 trabajos publicados en lengua inglesa, seleccionados por búsqueda en Medline (19661998) y en el International Pharmaceutical Abstracts (1970-1998) 22, de los cuales sólo 21 de los 95 fueron realizados en farmacias comunitarias, y que medían el impacto de servicios farmacéuticos sobre resultados en pacientes, concluyó que pocos estudios emplearon los diseños de investigación adecuados para controlar las amenazas a la validez interna y externa y que se deberían realizar proyectos que trataran de medir los tres tipos de variables ECHO (resultados económicos, clínicos y humanísticos) usando un diseño adecuado de investigación.

El Centro Cochrane publicó una revisión ${ }^{23}$, posteriormente puesta al $\mathrm{dia}^{24}$, que incluía trabajos recogidos en Medline, EMBASE, Pharmline, International Pharmaceutical Abstracts y otras, desde enero de 1966 a marzo de 1999, seleccionando 25 estudios. Se concluía que los estudios analizados apoyaban los papeles ampliados de los farmacéuticos en aconsejar a los pacientes y educación de los médicos. Sin embargo, las dudas acerca de la generabilidad de los estudios, la naturaleza pobremente definida de las intervenciones analizadas y la falta de estudios incluyendo valoración de costes y resultados en pacientes, indicaban que se necesitaba investigación más rigurosa para documentar los efectos de las intervenciones de los farmacéuticos sobre pacientes ambulatorios.

Se está construyendo evidencia de que la atención farmacéutica muestra un impacto sobre efectividades, calidad de vida y beneficios económicos. Se debería seguir investigando intensa y rigurosamente en atención farmacéutica para demostrar el valor para la sociedad de los servicios prestados por los farmacéuticos comunitarios.

También en este número de la Revista Española de Salud Pública se publican dos trabajos sobre estimación de la prevalencia de hipotiroidismo, uno en España y otro en Castilla y León, a partir del consumo de hormonas tiroideas. Debido a la falta de registros sobre enfermedades específicas, los estudios de utilización de medicamentos permiten, a partir del cálculo de las Dosis Diarias Definidas por 1.000 habitantes día (DHD) $)^{25-26}$ de determinados medicamentos 
marcadores, obtener una estimación de la prevalencia cruda de dichas enfermedades.

Como establece de Pedro ${ }^{27}$, para realizar este tipo de estudios es necesario, entre otros requisitos, identificar un marcador fácilmente disponible de frecuencia de, en este caso, hipotiroidismo en poblaciones; información de alta calidad sobre consumo de este marcador; buena disponibilidad de, en este caso, levotiroxina por la población; desarrollar, estandarizar y validar un modelo para medir prevalencia de, en este caso, hipotiroidismo usando este marcador; y estudiar la distribución geográfica de, en este caso, hipotiroidismo en España.

Es relevante destacar el incremento en utilización de hormona tiroidea en España que se ha producido en los dos períodos estudiados. Con relación a otro estudio realizado con datos de 1981-1982 el incremento sería diez veces mayor ${ }^{28}$. Mientras no tengamos mejores datos, la realización periódica de este tipo de estudios pueden servir para poner al día la prevalencia cruda de estas enfermedades, aunque se debería asegurar la validez del modelo.

Avorn y Soumerai ${ }^{29-31}$, a través de un ensayo controlado aleatorizado, midieron si las características del médico, tales como formación, edad, especialidad, práctica rural o urbana, etcétera, y la calidad y el número de exposiciones educativas, influían en la tasa de abandono de prescripción inapropiada. Concluyeron que la tasa de cambio en la prescripción era independiente de la mayor parte de las características estudiadas; los efectos de la intervención eran altamente significativos en 11 de los 14 subgrupos de médicos. Estos hallazgos documentaban que la educación cara a cara puede ser efectiva en mejorar la prescripción de una amplia variedad de médicos y que la brevedad, repetición, y reforzamiento de las prácticas recomendadas son componentes importantes en el diseño de tales programas.
El trabajo de Prieto Yerro y colaboradores que se publica en este número, siguiendo el modelo de Avorn y Soumerai ${ }^{29-32}$ de visita educativa, realizado en un hospital clínico y en un tipo de medicamento tan especializado, es relevante. El problema de duración de los efectos, como bien apunta la autora de acuerdo con estudios anteriores, implica un esfuerzo de continuidad.

El trabajo de Dacosta publicado en este número sobre medición de la performance de los servicios de suministros de establecimientos sanitarios, trataría de establecer indicadores para poder medir la eficiencia relativa de distintas unidades de producción a través de sus actividades o desempeño (performance). El sistema de medición, se indica que ha sido validado con la participación de 17 establecimientos sanitarios de Quebec (Canadá), presentando la proposición inicial de los indicadores para el nivel 1 y 2 . No está explícito si existe o prevé una proposición final, o bien una referencia al trabajo completo. La propuesta de Dacosta de extensión de este método a los hospitales españoles parece posible previo estudio de los modelos existentes, por lo menos en los servicios de farmacia hospitalaria ${ }^{33}$, por medio de una de las técnicas para evaluar eficiencia relativa, como puede ser el Análisis Envolvente de Datos (DEA, en su acronimia en ingles).

En el trabajo de Díaz Grávalos, publicado en este número, se estudia el conocimiento del precio de los fármacos por parte de los médicos de atención primaria de la provincia de Orense y la opinión de los mismos sobre la prescripción inducida, la industria farmacéutica y estrategias para contener el gasto. El conocimiento por el médico del precio de los medicamentos que se prescriben y la influencia que puede tener ese conocimiento en la prescripción ha sido un tema estudiado desde hace tiempo. En general, los médicos están predispuestos a ser conscientes del coste de sus prescripciones, pero les falta información sobre el precio de los medicamentos $y$, a menudo, hacen asunciones ine- 
xactas acerca del precio de los medicamentos prescritos ${ }^{34,35}$. En estudios anteriores también se detecta una tendencia hacia la subestimación de los precios de los medicamentos más caros, así como una sobrestimación de los precios de los medicamentos más baratos ${ }^{36}$. A pesar de ser consciente de ello, sorprende la gran influencia que tiene la industria farmacéutica, como se indica en el trabajo de Díaz Grávalos, y la tendencia a pasar el problema al paciente, como cuando se considera el copago como la medida más efectiva para la contención del gasto farmacéutico.

Lo importante para conseguir que se tengan en cuenta los aspectos relacionados con el gasto en medicamentos, es la consideración de que por muchos recursos que se destinen al ámbito sanitario las necesidades siempre serán mayores y por tanto es necesario ser eficiente (relación coste-beneficio) en el uso de estos y priorizar (coste-oportunidad, lo que se gaste en un apartado, en un tipo de enfermedad, en un paciente, no se va a poder gastar en otro). Esto a su vez implica más formación sobre evaluación económica, sobre farmacoeconomía, más disponibilidad de fuentes de información no interesadas de alta calidad, fácilmente accesibles y útiles, más disponibilidad de ayudas a la asistencia a congresos y reuniones por parte de las Administraciones Sanitarias, y más formación sobre ética.

Por último, el trabajo sobre resistencia a fluoroquinolonas en bacterias de origen animal publicado en este número, trae el tema de la gran amenaza que puede representar el paso por vía alimenticia de resistencias a los antibióticos desde los animales a los humanos, lo cual está en discusión. Como bien dicen los autores, la vigilancia de esta resistencia en animales y alimentos y el uso adecuado de estos antimicrobianos en animales es un tema importante en salud pública.

Todos los artículos comentados tienen una relación con la Salud Pública, por los métodos utilizados, por su influencia sobre el ecosistema en diversos aspectos, en fin por su influencia sobre la salud de la población. Se necesita estimular la investigación bien hecha sobre Salud Pública porque ésta, sin duda, va a beneficiar a la sociedad.

\section{BIBLIOGRAFÍA}

1. Hepler CD, Strand LM. Opportunities and responsabilities in pharmaceutical care. Am J Hosp Pharm 1990; 47: 533-43.

2. Strand LM, Cipolle RJ, Morley PC. Drug-related problems, their structure and function. DICP Ann Pharmacother 1990; 24: 1093-7.

3. Cipolle RJ, Strand LM, Morley PC. Pharmaceutical care practice. Minneapolis: Mc Graw-Hill; 1998.

4. Hepler CD, Grainger-Rousseau TJ. Pharmaceutical care versus traditional drug treatment. Is there a difference? Drugs 1995; 49: 1-10.

5. Tomechko MA, Strand LM, Morley PC, Cipolle RJ. Q and A from the pharmaceutical care project in Minnesota. Am Pharm 1995; NS35: 30-9.

6. Grainger-Rousseau TJ, Miralles MA, Hepler CD, Segal R, Doty RE, Ben-Joseph R. Therapeutic outcomes monitoring: application of pharmaceutical care guidelines to community pharmacy. J Am Pharm Assoc 1997; NS37(6): 647-61.

7. Herborg H, Soendergaard B, Froekjaer B, Fonnesbaek L, Jorgensen T, Hepler CD, et al. Improving drug therapy for patients with asthma -part 1: Patient outcomes. J Am Pharm Assoc 2001; 41(4): $539-50$

8. Herborg H, Soendergaard B, Jorgensen T, Fonnesbaek L, Hepler CD, Holst H, Froekjaer B Improving drug therapy for patients with asthma -part 2: Use of antiasthma medications. J Am Pharm Assoc 2001; 41(4): 551-9.

9. Narhi U, Airaksinen M, Tanskanen P, Erlund H Therapeutic outcomes monitoring by community pharmacists for improving clinical outcomes in asthma. J Clin Pharm Ther 2000; 25(3): 177-83.

10. Morck H. [Presentation of the results of the Therapeutic Outcomes Monitoring (TOM) study] Pharmazeutische-Zeitung 1996; 141(42): 55-8.

11. Varma S, McElnay JC, Hughes CM, Passmore AP, Varma M. Pharmaceutical care of patients with congestive heart failure: interventions and outcomes. Pharmacotherapy 1999; 19(7): 860-9. 
12. Skaer TL, Sclar DA, Markowski DJ, Won JKH. Effect of value-added utilities on prescription refill compliance and Medicaid health care expenditures - A study of patients with non-insulin-dependent diabetes mellitus. J Clin Pharm Ther 1993; 18(4): 295-9.

13. Van Veldhuizen MK, Widmer LB, Stacey SA, Popovich NG. Developing and implementing a pharmaceutical care model in an ambulatory care setting for patients with diabetes. Diabetes Educ 1995; $21: 117-23$.

14. Jaber LA, Halapy H, Fernet M, Tummalapalli S, Diwakaran H. Evaluation of a pharmaceutical care model on diabetes management. Ann Pharmacother 1996; 30: 238-43.

15. Erickson SR, Slaughter R, Halapy H. Pharmacists' ability to influence outcomes of hypertension therapy. Pharmacotherapy 1997; 17(1): 140-7.

16. Gourley DR, Gourley GA, Solomon DK, Portner TS, Bass GE, Holt JM, et al. Development, implementation, and evaluation of a multicenter pharmaceutical care outcomes study. J Am Pharm Assoc 1998; 38: 567-73.

17. Solomon DK, Portner TS, Bass GE, Gourley DR Gourley GA, Holt JM, et al. Clinical and economic outcomes in the hypertension and COPD arms of a multicenter outcomes study. J Am Pharm Assoc 1998; 38: 574-85.

18. Gourley GA, Portner TS, Gourley DR, Rigolosi EL, Holt JM, Solomon DK, et al. Humanistic outcomes in the hypertension and COPD arms of a multicenter outcomes study. J Am Pharm Assoc 1998; 38: 586-97.

19. Bluml BM, McKenney JM, Cziraky MJ. Pharmaceutical care services and results in project ImPACT: hyperlipidemia. J Am Pharm Assoc 2000; 40(2): 157-65.

20. Ellis SL, Carter BL, Malone DC, Billups SJ, Okano GJ, Valuck RJ, et al. Clinical and economic impact of ambulatory care clinical pharmacists in management of dyslipidemia in older adults: the IMPROVE study. Impact of Managed Pharmaceutical Care on Resource Utilization and Outcomes in Veterans Affairs Medical Centers. Pharmacotherapy 2000; 20(12): 1508-16.

21. Bernsten C, Bjorkman I, Caramona M, Crealey G, Frokjaer B, Grundberger E, et al. Improving the well-being of elderly patients via community pharmacy-based provision of pharmaceutical care: a multicentre study in seven European countries. Drugs Aging 2001; 18(1): 63-77.
22. Singhal PK, Raisch DW, Gupchup GV. The impact of pharmaceutical services in community and ambulatory care settings: evidence and recommendations for future research. Ann Pharmacother 1999; 33(12): 1336-55.

23. Bero LA, Mays NB, Barjesteh K, Bond C. Expanding the roles of outpatients pharmacists: effects on health services utilisation, costs, and patient outcomes (Cochrane Review). In: Cochrane Database Syst Rev 2000; (2): CD000336.

24. Beney J, Bero LA, Bond C. Expanding the roles of outpatient pharmacists: effects on health services utilisation, costs, and patient outcomes. Cochrane Database Syst Rev 2000; (3):CD000336. Review.

25. Bergman U, Grimsson A, Wahba AHW, Westerholm B, eds. Studies in drug utilization. Copenhagen: WHO Regional Office for Europe; 1979.

26. García Iñesta A. Estudios de utilización de medicamentos en España y análisis de la situación farmacoterapéutica. En: Instituto Nacional de la Salud. Estudios de utilización de medicamentos. Madrid: Instituto Nacional de la Salud; 1989. p.15-48.

27. Pedro Cuesta J de. Studies on the prevalence of paralysis agitans by tracer methodology. Karolinska Institute: Huddinge; 1986.

28. Barbeira Barja JM, García Iñesta A, Arias González A. Consumo de hormonas tiroideas como indicador de enfermedades tiroideas. Inf Ter Segur Soc 1983; 7: 232-41.

29. Avorn J, Soumerai SB. Improving drug-therapy decisions through educational outreach. A randomized controlled trial of academically based «detailing». N Engl J Med 1983; 308(24): 1457-63.

30. Soumerai SB, Avorn J. Economic and policy analysis of university-based drug «detailing». Med Care 1986; 24(4): 313-31.

31. Soumerai SB, Avorn J. Predictors of physician prescribing change in an educational experiment to improve medication use. Med Care 1987; 25(3): 210-21.

32. Soumerai SB, Avorn J. Principles of educational outreach («academic detailing») to improve clinical decision making. JAMA 1990; 263(4): 549-56.

33. Insalud. Guía para la evaluación y mejora de los servicios de farmacia hospitalaria. Madrid: Instituto Nacional de la Salud; 1999.

34. Reichert S, Simon T, Halm EA. Physicians' attitudes about prescribing and knowledge of the 
costs of common medications. Arch Intern Med 2000; 160(18): 2799-803.

35. Walzak D, Swindells S, Bhardwaj A. Primary care physicians and the cost of drugs: a study of prescribing practices based on recognition and in- formation sources. J Clin Pharmacol 1994; 34(12): 1159-63.

36. Glickman L, Bruce EA, Caro FG, Avorn J. Physicians' knowledge of drug costs for the elderly. J Am Geriatr Soc 1994; 42(9): 992-6. 